\title{
The Tunica Vaginalis Dorsal Graft Urethroplasty: Initial Experience
}

\author{
Roberto C. Foinquinos, Adriano A. Calado, Raimundo Janio, Adriana Griz, Antonio Macedo Jr, \\ Valdemar Ortiz
}

Division of Urology, State University of Pernambuco, Pernambuco, Brazil and Division of Urology, Federal University of Sao Paulo, Sao Paulo, Brazil

\begin{abstract}
Introduction: Nowadays, buccal mucosa grafts are the most successful method to reconstruct bulbar urethral strictures. Dorsal placement of the graft has been recently proposed, allowing the graft to be spread fixed on the tunica albuginea of the corporal bodies overlying the stricture. The dorsal graft is ingenious and represents a useful addition to the surgical armamentarium, since it offers a better chance for graft take than does the spongiosum when the urethra is diseased and poorly vascularized. We developed an additional reconstructive option using tunica vaginalis grafts, placed dorsally, for the treatment of anterior urethral strictures.

Surgical Technique: A total of 11 patients with anterior urethral strictures were treated with a tunica vaginalis graft urethroplasty. The surgical technique was done as described by Barbagli. The urethra was dissected from the corpora cavernosa and rotated 180 degrees. The dorsal urethral surface was exposed and fully opened. Both the distal and proximal lumina were calibrated. The tunica vaginalis graft was sutured, splayed and quilted over the corpora cavernosa using 6-0 PDS running stitches. The left side of the urethral mucosa was sutured to the graft using 6-0 PDS sutures. A 18F silicone Foley catheter was inserted at this point. The urethra was rotated back to its original position and sutured laterally to the right side of the graft. At the end of the procedure, the graft was completely covered by the urethra. With a follow-up ranging from 7 weeks to 5 months, all patients were voiding well (uroflowmetry $>14 \mathrm{~mL}$ per second).

Conclusion: This initial experience in 11 patients indicates that tunica vaginalis dorsal graft urethroplasty may be considered within the reconstructive armamentarium of genitourinary surgeons.
\end{abstract}

Key words: urethra; urethral stricture; urethroplasty; tunica vaginalis

Int Braz. J Urol. 2007; 33: 523-31

\section{INTRODUCTION}

Urethral reconstruction for trauma or stricture can require some of the most challenging techniques in urological surgery. Now that the role of urethrotomy has been drastically reduced, due to high long-term recurrence rates, urethroplasty is currently the best option to obtain a definitive cure for most urethral strictures (1).

Although an end-to-end anastomosis following resection of the diseased tissue is feasible for short localized strictures, additional tissue is often necessary for longer segments. Autologous nonurethral tissue grafts or flaps from genital and 
extragenital skin, bladder, rectal and buccal mucosa have been used.

Two major topics have been introduced in the past decade in urethral surgery in adults: the use of buccal mucosa and the dorsal approach for urethroplasty. The dorsal approach proposed by Barbagli has proven to be an effective and successful treatment of urethral stricture disease with little morbidity (2). On the other hand buccal mucosa grafting for urethroplasty of both urethral stricture and hypospadias repair has gained widespread acceptance during the past 10 years.

To explore the possibility of urethral reconstruction with a graft of tunica vaginalis to treat long strictures we previously reported the use of tunica vaginalis graft as a novel substitute for urethral reconstruction in rabbits before performing the operation in patients.

We present our short-term experience with tunica vaginalis grafts, placed dorsally, for the treatment of anterior urethral strictures. To our knowledge we report the first use of tunica vaginalis graft in urethroplasty.

\section{SURGICAL TECHNIQUE}

Preoperative evaluation included clinical history, physical examination, urine culture, uroflowmetry, and retrograde and voiding cystourethrography in all patients.

The surgical procedure was performed with the patient under epidural anesthesia. With the patient in the lithotomy position, through a perineal midline incision, the bulbocavernosus muscle was divided and the bulbar urethra exposed.

The urethra is freed from the bulbocavernous muscles for its entire length and the muscles are fixed to a retractor using four stitches. The bulbar urethra is dissected from the corpora cavernosa (Figure-1). The urethra is rotated $180^{\circ}$ and the distal extent of the stenosis is identified by gently inserting a $18 \mathrm{~F}$ catheter with a soft round tip until it meets resistance.

The dorsal urethral surface is incised in the midline until the catheter tip and urethral lumen are exposed (Figure-2). The stricture is then incised along its entire length by extending the urethrotomy both distally and proximally. Once the entire stricture has been incised, the length and width of the remaining urethral plate is measured.

The tunica vaginalis graft is trimmed to an appropriate size according to the length and width of the urethral defect (Figure-3). All harvests were performed using our standard technique. The graft was then defatted and kept in saline until it was ready to be placed on the recipient site.

The opened urethra is rotated onto the right side and the graft is sutured, splayed and quilted over the corpora cavernosa using 6-zero running stitches (Figure-4). The right urethral margin is sutured on the right side of the graft. The urethra is rotated over the graft and the left side of the graft is sutured to the left side of the urethra. At the end of the procedure the graft is completely covered by the urethra (Figure-5). A 18 Fr silicone catheter was inserted in the reconstructed urethra and urinary diversion was performed using a suprapubic catheter for 2 weeks. A nonadhesive compressive dressing was used and left in place for 3 days. Patients were mobilized on the first postoperative day and were discharged home 3 days after surgery. Transurethral micturition started after 2 weeks, when voiding cystography showed a patent urethra without extravasation.

This technique has so far been used in 11 patients. The etiology of the stricture was infective in 6 patients, iatrogenic in 3 and not known in 2 patients. The clinical details of the patients are given in the Table-1.

The mean age was 53.1 years (range 21 to 77 years). The median length of the stricture was 3.9 $\mathrm{cm}$ (range 2.3 to 8 ). No blood transfusion was required.

No periurethral leakage at voiding cystourethrogram was observed (Figure-6). None of the patients complained of postoperative testicular discomfort.

Early postoperative complication occurred in 1 patient undergoing tunica vaginalis urethroplasty. This patient had a small scrotal hematoma that resolved with drainage. We follow the patients using a standardized protocol, including a questionnaire on patient satisfaction and determination of flow rate. 


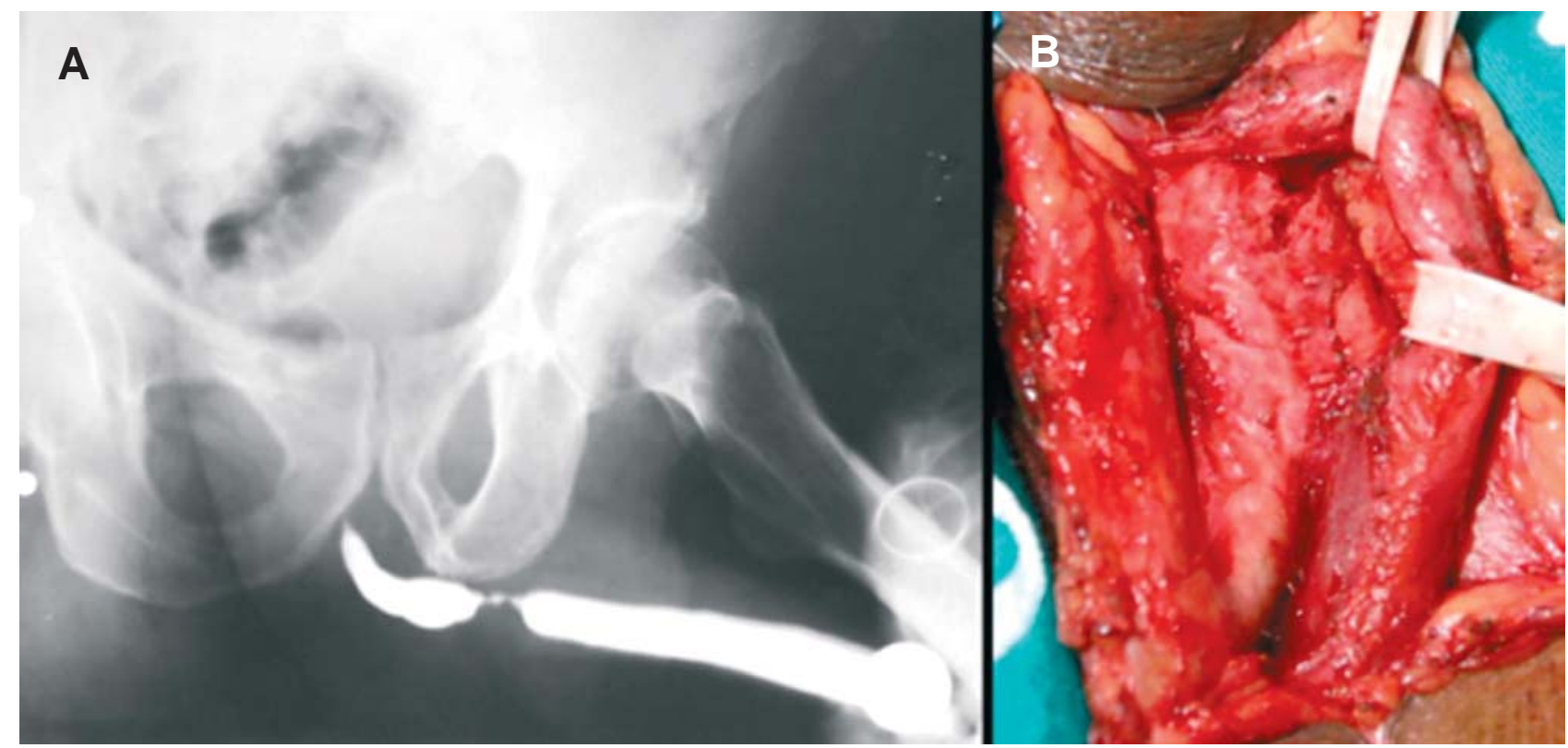

Figure 1 - A) Retrograde urethrography showing the urethral stricture. B) The bulbar urethra is completely mobilized from the corpora cavernosa.

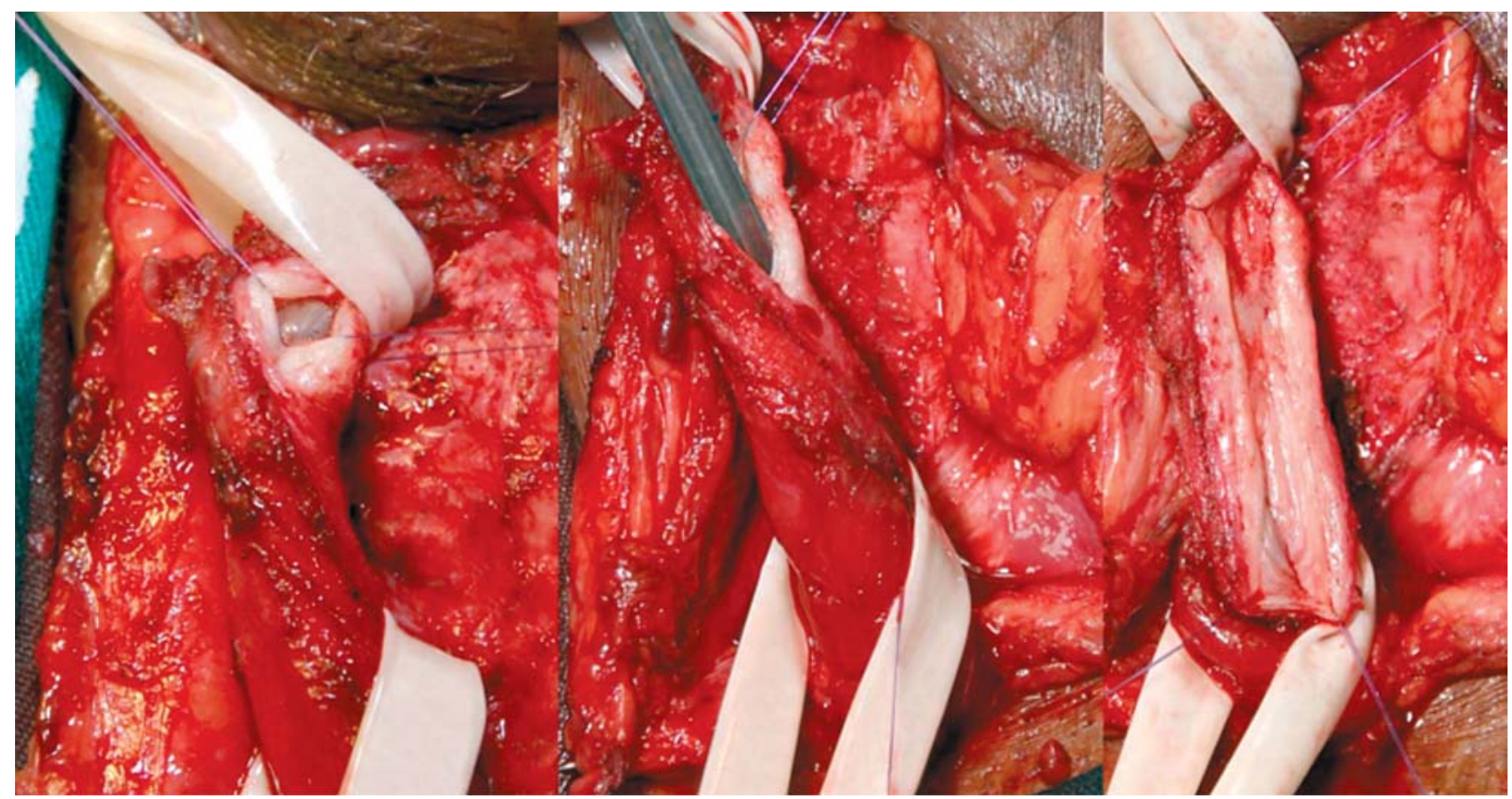

Figure 2 - The urethra is rotated 180 degrees, and the stricture is incised along its dorsal surface.

With follow-up ranging from 7 weeks to 5 months (mean follow-up 2.8 months), all patients were voiding well (uroflowmetry was more than $14 \mathrm{~mL}$ per second).
This study was approved by the Local Research Ethical Committee, reference number 025/ 2006. Informed consent was obtained from all patients. 


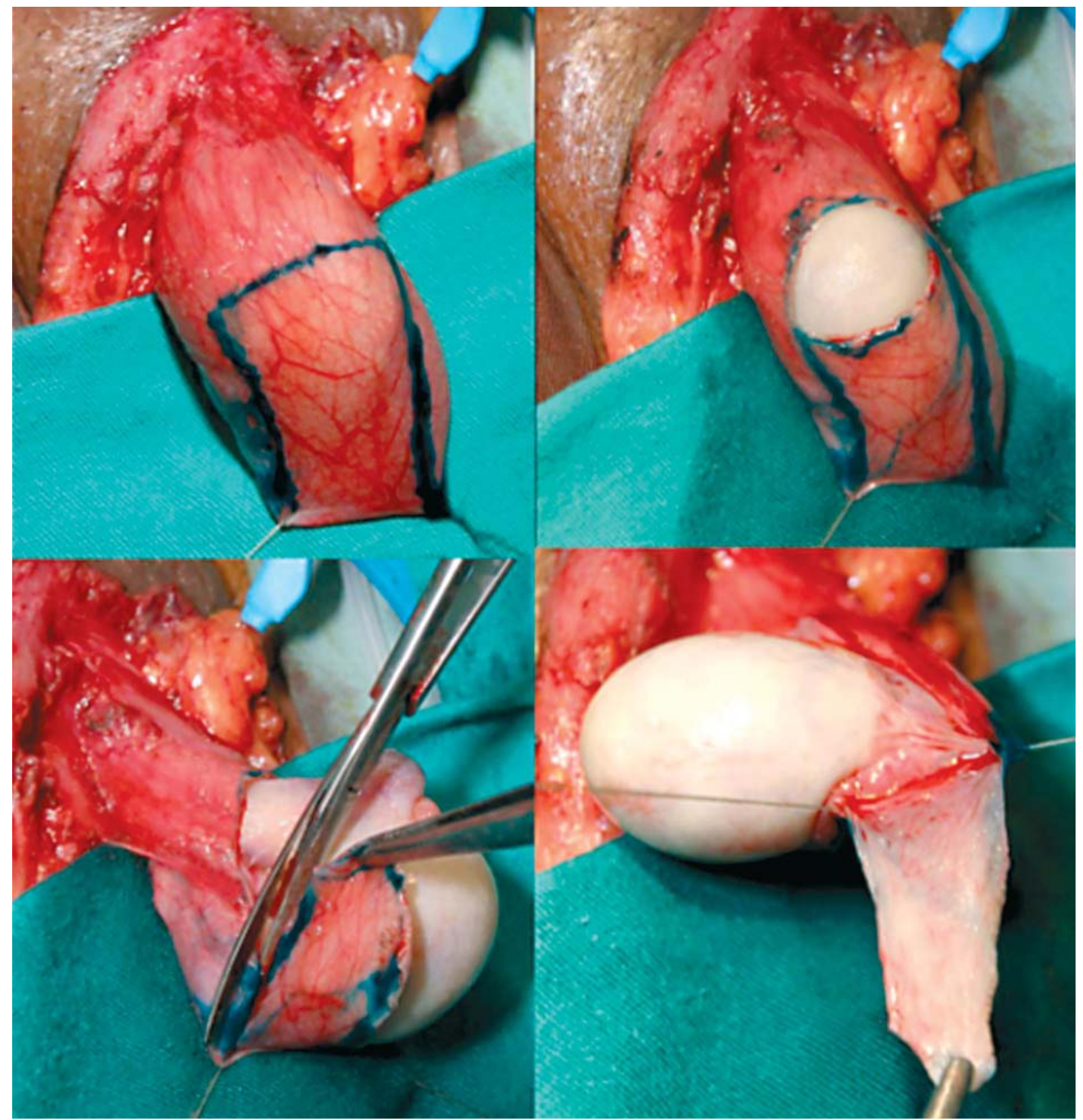

Figure 3 - The tunica vaginalis graft was obtained by sharp dissection with fine scissors.

\section{COMMENTS}

Surgery of the urethra for stricture disease is expanding because of the discouraging long-term high failure rate after urethrotomy. The use of new techniques and new urethral substitutes is challenging for the urologist.
In recurrent urethral strictures with failed prior internal urethrotomies, the indication is for an open approach. Similarly, urethroplasty may be the first option in long or multiple penile strictures, taking into account the high recurrence rates after internal urethrotomy. Although end-to-end anastomosis remains the method of choice in short uncomplicated 


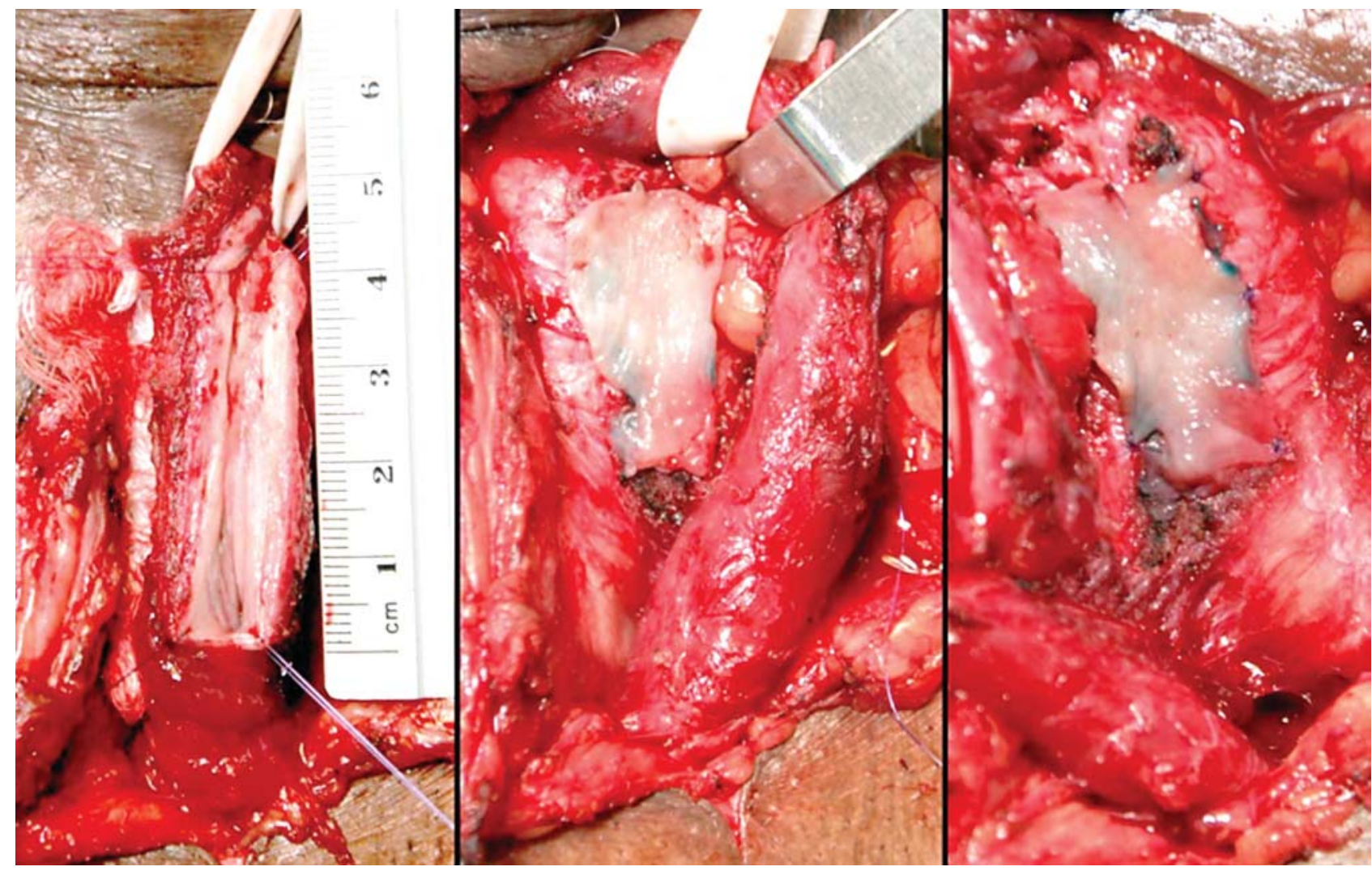

Figure 4-The tunica vaginalis graft was placed dorsally over the corpora cavernosa and tied with 6 running polydioxanone 6-0 sutures.

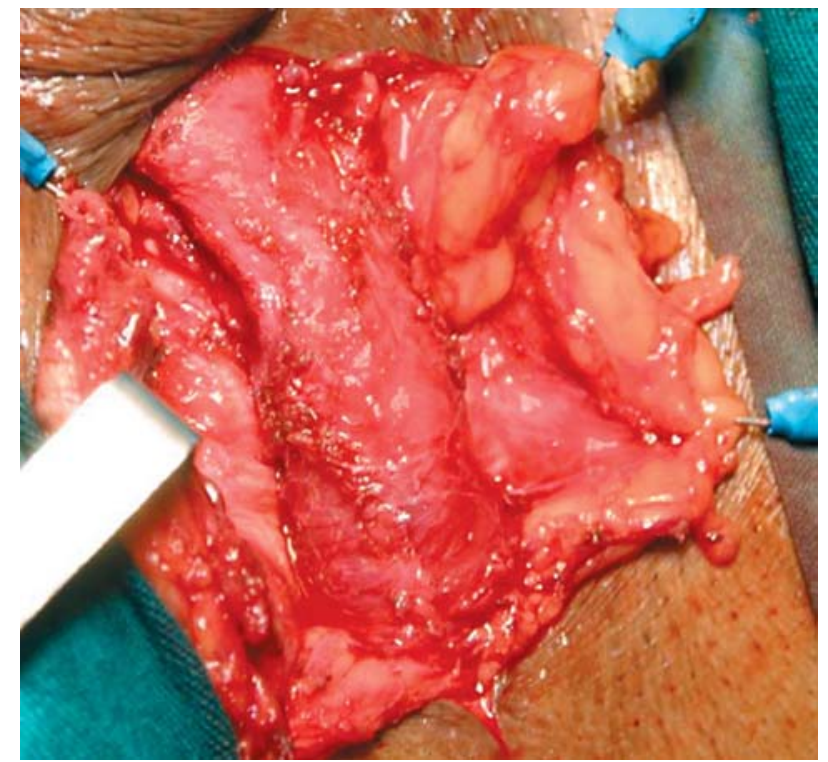

Figure 5 - Final aspect of the tunica vaginalis urethroplasty. The graft is completely covered by the urethra. strictures, patients with a stricture length exceeding 1 to $2 \mathrm{~cm}$ or with complex strictures, particularly in the penile area, require urethroplasty with either genital or extragenital graft material (1).

During the last 10 years buccal mucosal grafts have secured an important place in the armamentarium of substitution urethroplasties for the treatment of congenital and acquired anterior urethral disease (3). Advantages of buccal mucosal as a free graft are that it is hairless, and has a thick elastin rich epithelium making it tough and easy to handle, with a thin and highly vascular lamina propria that facilitates inosculation and imbibitions. Buccal mucosa is relatively easily harvested from the inner cheeks or lower lip with reputed minimal morbidity but oral complications have been reported in $0 \%$ to $8.3 \%$ of patients (4). Buccal mucosal grafts are currently the procedure of choice in treating bulbar urethral strictures not amenable to excision and primary end-to-end anastomosis. 
Table 1 - Clinical data of the patients.

\begin{tabular}{lccccc}
\hline Patient & $\begin{array}{c}\text { Age } \\
\text { (years) }\end{array}$ & $\begin{array}{c}\text { Stricture } \\
\text { Length }(\mathrm{cm})\end{array}$ & Etiology & Localization & $\begin{array}{c}\text { Previous } \\
\text { Treatments }\end{array}$ \\
\hline 1 & 77 & 5.0 & Iatrogenic & Bulbar & No \\
2 & 50 & 3.4 & Inflammatory & Bulbar & 2 UT, 1 D \\
3 & 61 & 8.0 & Iatrogenic & Bulbar & 1 UT \\
4 & 65 & 3.4 & Inflammatory & Penile & 2 UT \\
5 & 66 & 2.3 & Inflammatory & Penile & No \\
6 & 21 & 3.0 & Inflammatory & Bulbar & No \\
7 & 64 & 3.2 & Unknown & Bulbar & 1 UT, 1 D \\
8 & 49 & 3.4 & Inflammatory & Bulbar & 1 UT \\
9 & 25 & 3.0 & Inflammatory & Bulbar & No \\
10 & 37 & 4.0 & Unknown & Penile & No \\
11 & 69 & 4.8 & Iatrogenic & Penile & No \\
\hline
\end{tabular}

$U T=$ urethrotomies $; D=$ dilatations.
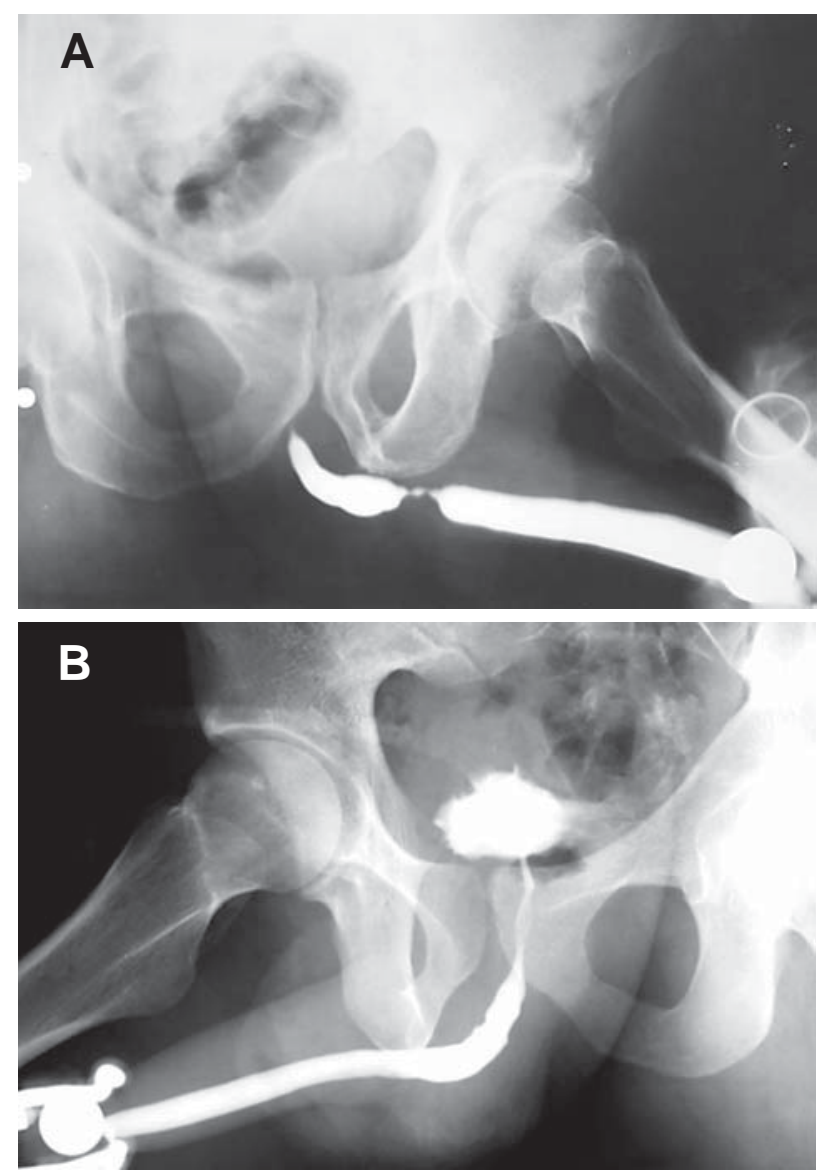

Figure 6-A) Voiding cystourethrogram showing a stricture. B) Voiding cystourethrogram after tunica vaginalis urethroplasty.
Barbagli et al. (2) popularized the concept of dorsal grafts anchored directly to the corpora, which has possible advantages compared to ventral graft urethroplasty that include better mechanical support, a better blood supply to the graft, and prevention of urethral diverticula. The Barbagli technique also has another advantage. The incision through the corpus spongiosum is through the thinnest and, therefore, least vascular part of the urethra, making bleeding substantially less than after ventral incision of the stricture.

Using an animal model we evaluated tunica vaginalis graft as a substitute for buccal mucosa in dorsal urethroplasty. All animals demonstrated a patent and functional urethra, as evidenced by radiographic and histological analyses. There was no evidence of infection or fistula (5).

Tunica vaginalis graft is much easier to harvest than other materials and their application is faster. In addition, the donor site is near and the tissue is abundant. Use of the tunica vaginalis graft has the potential to significantly decrease operative time. The reduced operative time has remarkable advantages and helps prevent troublesome complications from prolonged high lithotomy position.

We realize that the study have some weaknesses. The main one is that the study has a short follow-up. The aim of this study was not the com- 
parison of outcomes between penile skin, buccal mucosa and tunica vaginalis as substitute materials for urethroplasty. The present study only describes a new alternative in reconstructive urethral surgery and suggests the tunica vaginalis as the substitute material.

In our initial experience all patients had anatomically and functionally patent urethras as demonstrated by retrograde urethrography and uroflowmetry, but we know that the follow-up in this single study was brief. A wide series of patients with adequate follow-up are necessary to confirm our preliminary results obtained in this series.

\section{CONCLUSION}

This initial experience in 11 patients indicates that tunica vaginalis dorsal graft urethroplasty may be considered within the reconstructive armamentarium of genitourinary surgeons.

Correspondence address:

Dr. Adriano A. Calado

Rua Conselheiro Portela, 285 / 602

Recife, PE, 52020-030, Brazil

E-mail: caladourologia@yahoo.com.br

\section{EDITORIAL COMMENT}

The search for an ideal urethral substitute carries on as investigators continue to evaluate various materials for substitution urethroplasty. Buccal mucosa has proven to be a versatile substitute for strictures involving the meatus and the entire anterior urethra. The morbidity following buccal mucosa harvesting is mild and not cumbersome for patients. With increasing reports of the success of buccal mucosa in

\section{CONFLICT OF INTEREST}

None declared.

\section{REFERENCES}

1. Pansadoro V, Emiliozzi P: Which urethroplasty for which results? Curr Opin Urol. 2002; 12: 223-7.

2. Barbagli G, Selli C, di Cello V, Mottola A: A one-stage dorsal free-graft urethroplasty for bulbar urethral strictures. Br J Urol. 1996; 78: 929-32.

3. Dubey D, Kumar A, Mandhani A, Srivastava A, Kapoor R, Bhandari M: Buccal mucosal urethroplasty: a versatile technique for all urethral segments. BJU Int. 2005; 95: 625-9.

4. Dublin N, Stewart LH: Oral complications after buccal mucosal graft harvest for urethroplasty. BJU Int. 2004; 94: 867-9.

5. Calado AA, Macedo A Jr, Delcelo R, de Figueiredo LF, Ortiz V, Srougi M: The tunica vaginalis dorsal graft urethroplasty: experimental study in rabbits. J Urol. 2005; $174: 765-70$.

Accepted after revision:

December 30, 2006

urethroplasty, penile skin flaps are used more sparingly and the focus has shifted towards the use of free grafts. Newer urethral substitutes like colonic and tongue mucosa have been investigated as alternatives. The authors present results of a small study using the tunica vaginalis as a free graft on the urethra. There is experimental evidence to suggest that tunica vaginalis can be used successfully as a free graft for 
urethral strictures. It also appears to be a less morbid procedure than buccal mucosa urethroplasty. As the follow-up is short and number of patients limited, no firm conclusions can be drawn regarding the long term durability of this technique. However, the authors provide a strong case for comparison of this technique along with other substitution methods of urethroplasty in a larger number of patients.

\author{
Dr. Deepak Dubey \\ Assistant Professor, Dept. of Urology \\ Sanjay Gandhi Postgraduate Inst. Med. Sci. \\ Lucknow, India \\ E-mail:ddubey@sgpgi.ac.in
}

\section{EDITORIAL COMMENT}

The use of tunica vaginalis in reconstructive urethral surgery was mainly suggested by pediatric urologists in hypospadias repair. The objective was to provide vascular and mechanical support to the reconstructed urethra in order to avoid fistula formation. For our best knowledge, the authors of this manuscript reported, by the first, the use of tunica vaginalis graft in adult bulbar urethroplasty. The authors honestly emphasized the weaknesses and the drawbacks of their study, including a very short followup. It can be speculated that the success rate of this new kind of urethroplasty will probably decrease with extended follow-up; it is a "natural evolution" of any kind of urethroplasty apart from the substitute graft material. History of reconstructive urethral surgery is full of new and different substitute materials for urethral reconstruction, but, unfortunately, the final, long term follow-up outcome of any kind of urethroplasty is probably influenced more by the original urethral pathology than by the substitute material used for the repair.

Surgical treatment of urethral stricture diseases is a continually evolving process, and urologists have changed over time the substitute material. In the ' 90 s, skin grafts were the preferred substitute material for urethroplasty, but at present, many of us have left the use of skins graft. Up-to-date, buccal mucosa has become the most preferred substitute material in the treatment of urethral strictures as it is readily available in all patients and easily harvested from the inner cheek or lower lip and guarantees a concealed donor site scar and low oral morbidity. Buccal mucosa is hairless and has a thick elastin-rich epithelium, which makes it tough yet easy to handle, and a thin and highly vascular lamina propria, which facilitates inosculation and imbibition. These statements are now supported by literature evidence (1). In our experience, when we convey this message to the patients, they always appreciate it. Patients do not like to be considered as an experimental animal. For this reason, we are publishing all the results of our urethroplasties in an open and no-profit dedicate website (www.urethralcenter.it), to convey to the patients that the surgical technique we have selected for him is worldwide used in the urological community and the results of this technique are fully at his disposal. In the near future, every patient with a urethral stricture will be able to manage dedicated nomograms, which will predict his success and complication rate after surgery accurately on the basis of his age, of the site, of the length and the etiology of stricture.

This article is important for another reason. One of the basic principles in urethral reconstruction consists in the formation of an epithelialized tube from a buried strip of skin. In 1880, Duplay described a method for urethral construction in hypospadias, which was based on that principle, and reported the method that is usually associated with his name (2). In 1949, Denis Browne described a similar method for construction of the urethra in hypospadias (3). His method differs from Duplay's in a few essential respects. Over time, the Duplay's and Denis Browne's 
principles, according to which the buried strip of intact epithelium becomes an epithelialized tube, is widely exploited in reconstructive urology. In 1980, Monseur described the first dorsal urethroplasty and fully quoted the Duplay's principle: “...En premier lieu, l'urètre c'est un tube natural. Si une bandelette de peu ou de muqueuse enfouie, selon le principe de Duplay, tend spontanément à se tubuliser, combine plus facilement le tube urètral transfromé en bandelette par une incision longitudinal reprendera-t-il sa forme préalabe! Elargir le canal ne suffit pas, il faut en fixer les bords" (4). In 1996 (reference 2 in the text), we have fully exploited the Duplay's-Denis Browne's-Monseur's principles. The authors of this article showed in the experimental model and in humans that a buried strip of tunica vaginalis becomes an epithelialized tube. One hundred-twenty-seven years later, these authors confirm and expand the ingenious Duplay's principle: each strip of autologus epithelial tissue has the potential to be used for urethral regeneration. This is an important message for people involved in tissue engineering studies.
This new surgical technique should be now included in the armamentarium of the reconstructive urethral surgeon. The reconstruction of urethral channel is a challenging problem, for instance, sometimes, in our daily surgical practice, we might ask ourselves what kind of repair we are going to do in a particularly unusual and complex case. The replay should be "let us use the tunica vaginalis graft as suggested in the International Braz $\mathbf{J}$ Urol in 2007 !"

\section{REFERENCES}

1. Markiewicz MR, Lukose MA, Margarone JE, Barbagli G, Miller KS, Chuang SK: The oral mucosa graft: a systematic review. J Urol. 2007; 178: 387-94.

2. Duplay S: Sur le traitment chirurgical de l'hypospadias et de l'epispadias. Arch gen de méd. 1880; 145: 257-74.

3. Browne D: An operation for hypospadias. Proc Roy Soc Med. 1949; 42: 466-8.

4. Monseur J: L'élargissement de l'urètre au moyen du plan sus urètral. J Urol [Paris]. 1980; 86: 439-49.

Dr. Guido Barbagli \& Dr. Massimo Lazzeri Center for Reconstructive Urethral Surgery Arezzo, Italy E-mail: guido@rdn.it 